\title{
Low serum magnesium concentrations are associated with a high prevalence of premature ventricular complexes in obese adults with type 2 diabetes
}

\author{
Liana C Del Gobbo ${ }^{1 *}$, Yiqing Song ${ }^{2}$, Paul Poirier ${ }^{3}$, Eric Dewailly ${ }^{4}$, Ronald J Elin ${ }^{5}$ and Grace M Egeland ${ }^{1}$
}

\begin{abstract}
Background: Premature ventricular complexes (PVC) predict cardiovascular mortality among several adult populations. Increased arrhythmia prevalence has been reported during controlled magnesium (Mg) depletion studies in adults. We thus hypothesized that serum magnesium (sMg) concentrations are inversely associated with the prevalence of PVC in adults at high cardiovascular risk.

Methods: Anthropometric, demographic and lifestyle characteristics were assessed in 750 Cree adults, aged > 18 yrs, who participated in an age-stratified, cross-sectional health survey in Quebec, Canada. Holter electrocardiograms recorded heart rate variability and cardiac arrhythmias for two consecutive hours. Multivariate logistic regression was used to evaluate the associations between sMg and PVC.

Results: PVC prevalence in adults with hypomagnesemia ( $\mathrm{MMg} \leq 0.70 \mathrm{mmol} / \mathrm{L}$ ) was more than twice that of adults without hypomagnesemia ( $50 \%$ vs. $21 \%, p=0.015$ ); results were similar when adults with cardiovascular disease history were excluded. All hypomagnesemic adults with PVC had type 2 diabetes (T2DM). Prevalence of PVC declined across the sMg concentration gradient in adults with T2DM only ( $p<0.001$ for linear trend). In multivariate logistic regressions adjusted for age, sex, community, body mass index, smoking, physical activity, alcohol consumption, kidney disease, antihypertensive and cholesterol lowering drug use, and blood docosahexaenoic acid concentrations, the odds ratio of PVC among T2DM subjects with sMg $>0.70 \mathrm{mmol} / \mathrm{L}$ was 0.24 (95\% Cl: 0.06-0.98) $p=0.046$ compared to those with $\mathrm{sMg} \leq 0.70 \mathrm{mmol} / \mathrm{L}$.
\end{abstract}

Conclusions: $\mathrm{sMg}$ concentrations were inversely associated with the prevalence of PVC in patients with T2DM in a dose response manner, indicating that suboptimal sMg may be a contributor to arrhythmias among patients with T2DM.

Keywords: Magnesium, Hypomagnesemia, Premature ventricular complexes, Premature ventricular beats, Ectopic beats, Arrhythmia, Type 2 diabetes

\section{Background}

Premature ventricular complexes (PVC) are a relatively common electrocardiographic abnormality presenting in individuals with and without overt cardiovascular disease. In the latter case, PVC pathogenesis has traditionally been considered idiopathic and in the absence of

\footnotetext{
* Correspondence: liana.delgobbo@mail.mcgill.ca

'School of Dietetics \& Human Nutrition, McGill University, 21,111 Lakeshore Road, Ste. Anne de Bellevue, Quebec, H9X 3V9, Canada Full list of author information is available at the end of the article
}

severe clinical symptoms or structural cardiac abnormalities, their presence benign $[1,2]$. Recent prospective studies evaluating the prognostic significance of PVC for sudden and total cardiac death in apparently healthy adults directly challenge this view. Among individuals without history of heart disease or stroke, PVC counts independently predicted future cardiac events or sudden cardiac death compared to those without PVC [3-7]. These findings are consistent with hypothesis that PVC may indicate electrical instability and increased

\section{C) Biomed Central}


susceptibility to ventricular fibrillation [6], the most common cause of sudden cardiac death.

$\mathrm{Mg}^{2+}$ antagonizes calcium on the atrioventricular node [8] and myocardial $\mathrm{Mg}^{2+}$ deficiency decreases intracellular potassium, resulting in a less negative resting membrane potential and enhanced vulnerability to ventricular arrhythmia [9-11]. Two randomized, doubleblind crossover studies by USDA scientists showed that reducing intake of dietary magnesium $(\mathrm{Mg})$ in postmenopausal women in a metabolic unit to $33-50 \%$ of the $\mathrm{Mg}$ recommended dietary allowance can induce heart rhythm changes [12,13]. During the Mg depletion phase, calcium, potassium, copper and other nutrients were concomitantly supplemented [12] and arrhythmias were relieved by $\mathrm{Mg}$ supplementation, suggesting that $\mathrm{Mg}$ insufficiency resulting from a diet that would not be considered an atypical Western menu can induce arrhythmias in older women.

In adults from the Framingham Offspring cohort free of clinically apparent heart disease, total serum magnesium $(\mathrm{sMg})$ was significantly inversely associated with prevalence of complex or frequent PVC after adjustment for multiple covariates, including serum potassium [14]. As type 2 diabetes (T2DM) is the most common condition associated with low sMg or hypomagnesemia ( $\mathrm{sMg}$ $\leq 0.70 \mathrm{mmol} / \mathrm{L}$ ) [15] and T2DM significantly increases risk of PVC and sudden cardiac death [16], the association between sMg and PVC may be modified by diabetic status. However, testing for such potential effect modification has not been reported previously.

We hypothesized that $\mathrm{sMg}$ concentrations are inversely associated with the prevalence of PVC in a general adult population at high cardiovascular risk, James Bay Cree, and tested for effect modification by T2DM. Mg intakes in Cree adults, particularly Cree men, are inadequate, with $93 \%$ of men $19-30$ yrs and $100 \%$ of men $\geq$ 31 yrs consuming less than the $\mathrm{Mg}$ recommended dietary intake in some communities [17]. Due to rapid dietary and lifestyle transition and consequent increasing obesity rates, prevalence of T2DM among Cree is among the highest in the world [18].

\section{Methods}

A subset of adults ( $\geq 18 \mathrm{yrs}$ ) of Cree descent from the James Bay region of Quebec, Canada were selected from a comprehensive aboriginal health survey 'Nituuchischaayihitaau Aschii: A Multi-Community Environment and Health Longitudinal Study in Iiyiyiu Aschii.' This cross-sectional study included fasting participants from seven communities sampled between 2005-2009 for which sMg, covariates and 2-hr Holter electrocardiogram recordings were available. Bilingual Cree were trained to conduct interviews and recruit participants. Ethics for the health survey was obtained from the Cree
Board of Health and Social Services of James Bay (CBHSSJB), Centre Hospitalier Universitaire de Québec (Laval), and McGill universities. A research agreement with communities was developed with the Cree Board of Health and all individual study participants provided informed written consent in Cree or English.

A total of 834 adults were assessed for anthropometric measures, demographic and lifestyle variables (smoking, alcohol consumption, exercise); sMg, full medical history, and Holter data (PVC) were available for 750 adults included in analysis. Individuals with missing values did not differ significantly in covariate distribution from included individuals.

\section{Variable assessment}

Anthropometric variables were measured without shoes by trained nurses. Height, measured in centimeters using a graduated tape, and weight, determined using a foot-to-foot bioelectrical impedance instrument (Tanita Corp, Arlington Heights, IL, USA), were combined to obtain body mass index (BMI). Exercise (frequency in which individuals reported to engage in vigorous physical activity for at least $20 \mathrm{~min}$ at a time) was assessed by a culturally adapted, short version of the International Physical Activity Questionnaire validated in Cree adults [19]. Smoking status (current, former or never) and alcohol consumption ( $\geq 3$ drinks/day, $<3$ drinks day, or never, of standard drinks of beer, liquor, wine, mixed drinks, or shooters) were self-reported. As cellular and animal models, epidemiological studies and clinical trials suggest that long-chain polyunsaturated fatty acids exert anti-arrhythmic effects $[20,21]$, we tested the potentially modifying effects of plasma eicosapentaenoic acid (EPA) and docosahexaenoic acid (DHA) as nutritional covariates in models. Medical charts for each participant were reviewed and detailed cardiovascular event history or conditions, metabolic conditions, cardiovascular drugs/ supplement use, and selected other conditions were recorded. In characterizing the metabolic syndrome (MetS), the consensus definition was used [22]. Adults with T2DM had previously been diagnosed or presented with fasting glucose (FG) concentrations $\geq 7 \mathrm{mmol} / \mathrm{L}$.

Serum $\mathrm{Mg}$ was determined at Trace Elements Laboratory the London Laboratory Services group, London, Ontario, Canada using the colorimetric endpoint method with addition of xylidyl blue. Mg content was measured photometrically as a function of the decrease in xylidyl blue absorbance (laboratory reference range: 0.65-1.05 mmol/L). For the determination of EPA and DHA concentrations, $200-\mu \mathrm{L}$ aliquots of plasma were extracted after the addition of chloroform: methanol (2:1), in the presence of a known amount of internal standard (diheptadecanoyl phospholipid). Total phospholipids were isolated from the lipid extract via thin- 
layer chromatography with the use of developing solvent heptane:isopropyl ether:acetic acid (60:40:3). Transmethylation with boron trifluoride and methanol preceded capillary gas-liquid chromatography to determine fatty acid concentrations.

Frequency of premature ventricular beats, distinct from supraventricular beats, were derived from a 2-hour Holter monitoring system (GE Marquette Series 8500) with a recording frequency of $128 \mathrm{~Hz}$. Seven leads (derivations $\mathrm{V} 5, \mathrm{~V} 1$, and $\mathrm{AVF}$ ) were installed when subjects arrived to the clinic. During the 2-hour recording, subjects remained at the clinic, completing anthropometric measurements and lifestyle questionnaires.

\section{Statistical approach}

PVC prevalence for all adults $\geq 18$ yrs $(\mathrm{n}=750)$ on 2 hour Holters was presented stratified by demographic, anthropometric and lifestyle variables. We considered $\geq$ $1 \mathrm{PVC} / \mathrm{hr}$ and $>6 \mathrm{PVC} / \mathrm{hr}$ recording as endpoints in this high cardiometabolic risk sample; elevated risk for sudden cardiac death was reported for high-risk participants in the Cardiovascular Health Study with $>153$ PVC over $24 \mathrm{~h}$, or about 6 events/hr [23].

Unadjusted prevalence of PVC across sMg groupings for the entire sample and subgroups (with cardiovascular event history or conditions excluded, those with T2DM, and MetS) was determined. Frequencies were compared using $X^{2}$ tests or Fisher's exact test when sample sizes in contingency tables were small. Odds ratios and $95 \%$ confidence intervals using multivariate logistic regression for $\geq 1 \mathrm{PVC} / \mathrm{hr}$ and $>6 \mathrm{PVC} / \mathrm{hr}$ across the sMg concentration range for the whole population were determined for five different covariate models. Hypomagnesemia was defined as $\mathrm{sMg} \leq 0.70 \mathrm{mmol} /$ L. In subgroup analysis for adults with type 2 diabetes and no prior cardiovascular disease (CVD) $(n=149)$, only the odds ratio of $\geq 1 \mathrm{PVC} / \mathrm{hr}$ for normomagnesemic diabetics $(\mathrm{sMg}>0.70 \mathrm{mmol} / \mathrm{L})$ relative to the reference group of hypomagnesemic diabetics $(\mathrm{sMg} \leq 0.70$ $\mathrm{mmol} / \mathrm{L}$ ) were determined due to sample size constraints. For multivariate models, individuals with prior CVD were defined as those with one or more condition listed under "cardiovascular event history or conditions" or taking CVD drugs in Table 1; hypertensives were excluded from this definition. Additional sensitivity analysis was performed excluding adults with kidney disease, as this condition is associated with altered $\mathrm{Mg}$ homeostasis and sMg concentrations [24].

\section{Results}

Our population included 441 women (58.8\%) and 309 men $(41.2 \%)$ with a mean age of $38.9 \pm 14.8$ yrs. Mean BMI was $33.9 \pm 7.0 \mathrm{~kg} / \mathrm{m}^{2}$. More than half of adults (51.3\%) identified as current smokers. Hypertension was
Table 1 Medical chart review of adults ( $\geq 18 \mathrm{yrs}$ ) of the general Cree population $(\mathbf{n}=\mathbf{7 5 0})$

\begin{tabular}{|c|c|}
\hline Cardiovascular event history or conditions & N \\
\hline Ischemia/infarction & 2 \\
\hline Cardiac insufficiency & 4 \\
\hline Atrial fibrillation & 3 \\
\hline Ventricular arrhythmia/tachycardia & 5 \\
\hline Bradycardia & 3 \\
\hline Ventricular hypertrophy & 2 \\
\hline Angina & 6 \\
\hline Hypertension (diagnosed) & 185 \\
\hline (BP > 130/85 mmHg, or treatment of diagnosed) & 249 \\
\hline Ischemic heart disease & 6 \\
\hline Diseases of the pulmonary circulation & 3 \\
\hline Stroke & 4 \\
\hline Cerebrovascular diseases & 11 \\
\hline Diseases of arteries, arterioles or capillaries & 6 \\
\hline \multicolumn{2}{|l|}{ Cardiometabolic conditions } \\
\hline Metabolic syndrome & 352 \\
\hline (excluding diagnosed type 2 diabetics/FG $\geq 7 \mathrm{mmol} / \mathrm{L}$ ) & 140 \\
\hline Gestational diabetes (history of) & 23 \\
\hline Type 1 diabetes & 1 \\
\hline Type 2 diabetes (diagnosed) & 140 \\
\hline (diagnosed or $F G \geq 7 \mathrm{mmol} / \mathrm{L}$ ) & 176 \\
\hline \multicolumn{2}{|l|}{ Cardiovascular drug/supplement use } \\
\hline Antiplatelet & 6 \\
\hline Antihypertensives & 183 \\
\hline Diuretics (specifically) & 62 \\
\hline Cholesterol-lowering drugs & 79 \\
\hline Statins (specifically) & 73 \\
\hline Multivitamins & 41 \\
\hline Mg supplements & 8 \\
\hline \multicolumn{2}{|l|}{ Hormonal or menstrual status } \\
\hline Post-menopausal & 104 \\
\hline Hormone replacement therapy & 1 \\
\hline Oral contraceptives & 92 \\
\hline Currently breastfeeding & 35 \\
\hline \multicolumn{2}{|l|}{ Other } \\
\hline Kidney disease & 13 \\
\hline Cancer & 6 \\
\hline
\end{tabular}

the most common CVD condition (33.2\%) and antihypertensive drugs the most common class of CVD medications taken $(24.4 \%)$. The percentage of adults with MetS was 46.9\%; prevalence of T2DM (diagnosed or FG $\geq 7 \mathrm{mmol} / \mathrm{L}$ ) was $23.5 \%$ (Table 1 ).

Prevalence and frequency of PVC differed by smoking status and was significantly higher in men, older participants, those with MetS or T2DM $(p<0.001$ for each covariate). PVC prevalence varied significantly with alcohol consumption, exercise and CVD history (excluding hypertensives) $(p<0.05)$. There was a trend towards an association between PVC and hypertension $(p<0.10)$ 
but obesity was not significantly associated with PVC ( $p$ $=0.39)$ (Table 2) nor was diuretics use $(p=0.63)$.

Unadjusted prevalence of PVC in adults with hypomagnesemia $(\mathrm{sMg} \leq 0.70 \mathrm{mmol} / \mathrm{L})$ was over twice that of adults without hypomagnesemia ( $50 \%$ vs. $21 \%, p=$ $0.015)$; results were not different when adults with cardiovascular disease history were excluded (Figure 1). In multivariate logistic regression analyses for all adults ( $\mathrm{n}$ = 750), odds of $\geq 1 \mathrm{PVC} / \mathrm{hr}$ declined precipitously from the hypomagnesemic reference group to 0.24 (95\% CI: $0.07-0.87)$ in those with $\mathrm{sMg} 0.71-0.80 \mathrm{mmol} / \mathrm{L}$ in the fully adjusted model (Model 5); risk did not substantively change at higher sMg concentrations (Table 3 ). Due to low sample size among the hypomagnesemic reference group, it was not possible to further define the sMg threshold associated with reduced odds $\geq 1$ PVC in this sample. Model covariates had little influence on

\begin{tabular}{|c|c|c|c|}
\hline Characteristic & $\begin{array}{l}\text { No PVC } \\
\mathrm{n}=591\end{array}$ & $\begin{array}{c}1-6 \mathrm{PVC} / \mathrm{hr} \\
\mathrm{n}=118\end{array}$ & $\begin{array}{c}>6 \mathrm{PVC} / \mathrm{hr} \\
\mathrm{n}=41\end{array}$ \\
\hline \multicolumn{4}{|l|}{ Sex } \\
\hline Male & 217 (37\%) & 64 (54\%) & $28(68 \%)$ \\
\hline Female & $374(63 \%)$ & $54(46 \%)$ & $13(32 \%)^{* * *}$ \\
\hline \multicolumn{4}{|l|}{ Age (yrs) } \\
\hline $18-30$ & $194(33 \%)$ & $23(19 \%)$ & $5(12 \%)$ \\
\hline $30-50$ & 302 (51\%) & $44(37 \%)$ & 14 (34\%) \\
\hline$\geq 50$ & 95 (16\%) & $51(43 \%)$ & $22(54 \%)^{* * *}$ \\
\hline \multicolumn{4}{|l|}{ BMI $\left(\mathrm{kg} / \mathrm{m}^{2}\right)$} \\
\hline$<30$ & 170 (29\%) & $36(31 \%)$ & $8(20 \%)$ \\
\hline$\geq 30$ & $421(71 \%)$ & $82(69 \%)$ & 33 (80\%) \\
\hline \multicolumn{4}{|l|}{ Smoking } \\
\hline Current & $329(56 \%)$ & 40 (34\%) & 16 (39\%) \\
\hline Former & 204 (34\%) & $61(52 \%)$ & 21 (51\%) \\
\hline Never & $58(10 \%)$ & $17(14 \%)$ & $4(10 \%)^{* * *}$ \\
\hline \multicolumn{4}{|l|}{ Alcohol $^{1}$} \\
\hline$<3$ drinks/d & $271(46 \%)$ & $48(41 \%)$ & $10(24 \%)$ \\
\hline Never & 312 (54\%) & 69 (59\%) & $31(76 \%)^{* *}$ \\
\hline \multicolumn{4}{|l|}{ Exercise } \\
\hline$\geq 4$ times/wk & 187 (32\%) & 37 (31\%) & 13 (32\%) \\
\hline 1-3 times/wk & 217 (36\%) & $32(27 \%)$ & $10(24 \%)$ \\
\hline None & 187 (32\%) & 49 (42\%) & $18(44 \%)^{* *}$ \\
\hline Hypertension & $153(26 \%)$ & 41 (35\%) & $15(37 \%)^{*}$ \\
\hline CVD history ${ }^{1}$ & $18(3 \%)$ & $7(6 \%)$ & $4(10 \%)^{* *}$ \\
\hline MetS $S^{2}$ & 95 (16\%) & $30(25 \%)$ & $15(37 \%)^{* * *}$ \\
\hline Diabetes $^{3}$ & 122 (21\%) & $37(31 \%)$ & $17(41 \%)^{* * *}$ \\
\hline
\end{tabular}

${ }^{*} p<0.01{ }^{* *} p<0.05{ }^{* * *} p<0.001$

${ }^{1}$ Includes history of cardiovascular events/arrhythmia or CVD, excludes hypertensives

${ }^{2}$ Excludes diagnosed type 2 diabetics $/ \mathrm{FG} \geq 7 \mathrm{mmol} / \mathrm{L}$

${ }^{3}$ Includes diagnosed type 2 diabetes or $\mathrm{FG} \geq 7 \mathrm{mmol} / \mathrm{L}$ adjusted odds ratios; inclusion of EPA in any model had no impact on estimates and was excluded.

Significantly decreased adjusted odds of $>6 \mathrm{PVC} / \mathrm{hr}$ were observed in those with sMg above a sMg 0.75 $\mathrm{mmol} / \mathrm{L}$ threshold in the whole population for all covariate models (Additional file 1). Odds of $>6 \mathrm{PVC} / \mathrm{hr}$ for those with sMg 0.75-0.80 relative to those with < $0.75 \mathrm{mmol} / \mathrm{L}$ were 0.10 (95\% CI: $0.02-0.46)$ in the fully adjusted model (Model 5). A sMg threshold of 0.75 $\mathrm{mmol} / \mathrm{L}$ was selected for this endpoint because ORs were maximally reduced using this cutoff value (Additional file 2).

Further evaluation of the characteristics of hypomagnesemic subjects in this population revealed that all individuals with PVC had T2DM. When participants were stratified by diabetic status, prevalence of PVC declined across the sMg concentration gradient in adults with T2DM only $(p<0.001)$ (Figure 2$)$. Decline in PVC prevalence with increasing $\mathrm{sMg}$ among participants with T2DM was significant even when hypomagnesemic adults were excluded $(p=0.005)$. Addition of diabetic medication use (metformin, gluconorm, or insulin) as covariates had no influence on model estimates. While only $1 \%$ of subjects without T2DM were hypomagnesemic $(n=5)$, none of these subjects had PVC during recording. In the subgroup with MetS but without T2DM, no significant difference in PVC prevalence across the sMg concentration gradient was observed ( $p$ $=0.84$ ).

Among adults with T2DM and no CVD history ( $\mathrm{n}=$ 149), individuals without hypomagnesemia $(\mathrm{sMg}>0.70$ $\mathrm{mmol} / \mathrm{L}$ ) had significantly lower risk of PVC on 2-hr Holter recording in fully adjusted models [OR 0.24 (95\% CI: 0.06-0.98)] (Table 4). Sensitivity analysis excluding remaining individuals with kidney disease $(n=4$ without CVD history) had no influence on model estimates.

\section{Discussion}

Hypomagnesemia \& ventricular arrhythmias

Our finding of significantly elevated PVC prevalence in adults with hypomagnesemia relative to adults without reduced sMg is consistent with early clinical studies reporting ventricular fibrillation and tachycardia in hypomagnesemia [25,26]. Hypomagnesemia is an established risk factor for polymorphic ventricular tachycardia torsades de pointes [27] and intravenous magnesium sulfate remains first-line therapy for tosades de pointes associated with long QT interval according to current ACC/AHA/ESC guidelines [28]. It has been suggested that the trigger for torsades de pointes are PVC resulting from an early afterdepolarization generated during the abnormally prolonged repolarization phase of affected myocardium [29]. 


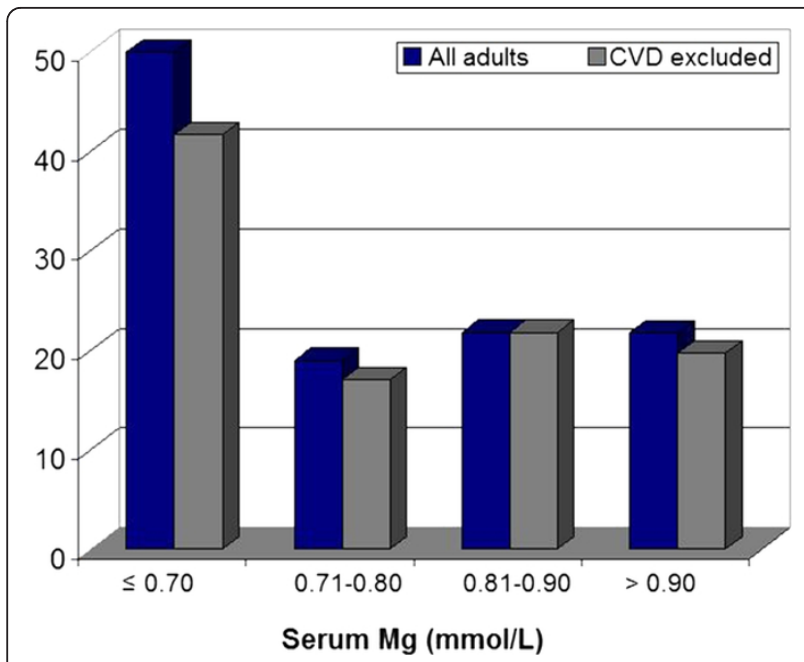

Figure 1 PVC prevalence (\%) during 2-hr Holters among adults $(\mathrm{n}=\mathbf{7 5 0})$ according to $\mathrm{sMg}$ concentrations $(\mathrm{mmol} / \mathrm{L})^{1}$. Legend: CVD excluded (grey bars) omits individuals with cardiovascular event history or conditions. Difference in PVC prevalence among participants with $\mathrm{sMg} \leq 0.70 \mathrm{vs}$. $>0.70 \mathrm{mmol} / \mathrm{L}$ among all adults (blue bars): $p=0.015$; for CVD excluded (grey bars): $p=0.07$.

Reports of increased prevalence of ventricular arrhythmias in individuals with low sMg concentrations have been criticized as lacking in evidence that the effect is attributable to $\mathrm{Mg}$ alone as opposed to a combination of hypomagnesemia and hypokalemia [30]. As we did not measure serum potassium in our cross-sectional design, this study provides no evidence to rebuke this claim, particularly as hypomagnesemia and hypokalemia frequently coexist and have similar etologies. In the Framingham Offspring cohort, apparently the only other general population study associating sMg with PVC, multivariate logistic regression analyses revealed that both serum potassium and Mg concentrations, when

Table 3 Adjusted odds ratios \& $95 \%$ confidence intervals for presence of $\geq 1$ PVC on 2-hr Holters across the sMg concentration range $(n=750)$

\begin{tabular}{ccccc}
\hline $\begin{array}{c}\text { sMg } \\
\text { (mmol/L) }\end{array}$ & $\mathbf{5 0 . 7}$ & $\mathbf{0 . 7 1 - 0 . 8 0}$ & $\mathbf{0 . 8 1 - 0 . 9 0}$ & $>\mathbf{0 . 9 0}$ \\
\hline Model 1 & 1.00 & $0.26(0.07-0.88)$ & $0.27(0.08-0.93)$ & $0.22(0.06-0.81)$ \\
\hline Model 2 & 1.00 & $0.25(0.07-0.85)$ & $0.26(0.08-0.88)$ & $0.19(0.05-0.71)$ \\
\hline Model 3 & 1.00 & $0.25(0.07-0.85)$ & $0.26(0.08-0.88)$ & $0.20(0.05-0.73)$ \\
\hline Model 4 & 1.00 & $0.24(0.07-0.86)$ & $0.25(0.07-0.92)$ & $0.19(0.05-0.77)$ \\
\hline Model 5 & 1.00 & $0.24(0.07-0.87)$ & $0.26(0.07-0.93)$ & $0.18(0.04-0.73)$ \\
\hline
\end{tabular}

Model 1: adjusted for age and sex

Model 2: additionally adjusted for region and BMI

Model 3: additionally adjusted for smoking, physical activity, alcohol consumption

Model 4: additionally adjusted for history of cardiovascular events/disease, type 2 diabetes, kidney disease, antihypertensive and cholesterol lowering drug use

Model 5: additionally adjusted for blood DHA

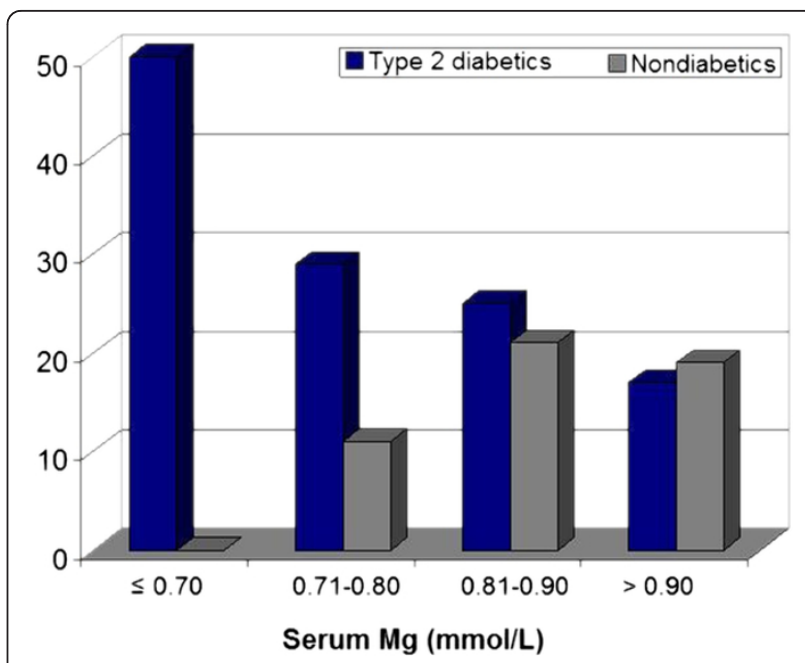

Figure 2 PVC prevalence (\%) during 2-hr Holters among adults without CVD $(\mathrm{n}=652)$ by $\mathrm{sMg}$ concentrations $(\mathrm{mmol} / \mathrm{L})$ and diabetic status ${ }^{1}$. Legend: PVC prevalence declined with $\mathrm{sMg}$ concentration in adults with T2DM (blue bars) only ( $p<0.001$ for trend), even when hypomagnesemic adults were excluded ( $p=$ 0.005 for trend). While no nondiabetic (grey bars) had PVC during monitoring, only $1 \%$ of nondiabetics were hypomagnesemic (sMg $\leq$ $0.70 \mathrm{mmol} / \mathrm{L})$

simultaneously entered into models, were inversely associated with complex or frequent PVC occurrence $(p<$ $0.04)$. Each standard deviation decrement in potassium $(0.48 \mathrm{mmol} / \mathrm{L})$ or $\mathrm{Mg}(0.08 \mathrm{mmol} / \mathrm{L})$ was associated with a similar increase in odds PVC [27\% (95\% CI 6$51 \%$ ) for potassium, and $20 \%$ (95\% CI 3-41\%) for $\mathrm{Mg}$ ] [14]. From physiologic studies, $\mathrm{Mg}$ is an established regulator of major ion channels in the cardiovascular system, including potassium, calcium, sodium, and others [31]. Potassium is not effective in the suppression of cardiac arrhythmia occurring in the presence of normal

Table 4 Effect of hypomagnesaemia in type 2 diabetics without CVD history on adjusted odds ratios \& $95 \%$ confidence intervals for $\geq 1$ PVC on 2-hr Holters ( $n=$ 149)

\begin{tabular}{ccc}
\hline & $\begin{array}{c}\text { Type 2 diabetics } \\
\mathbf{s M g} \leq \mathbf{0 . 7 0} \mathbf{~ m m o l} / \mathbf{L}\end{array}$ & $\begin{array}{c}\text { Type 2 diabetics } \\
\mathbf{s M g}>\mathbf{0 . 7 0} \mathbf{~ m m o l} / \mathbf{L}\end{array}$ \\
\hline Model 1 & 1.00 & $0.28(0.08-0.98)$ \\
\hline Model 2 & 1.00 & $0.25(0.07-0.92)$ \\
\hline Model 3 & 1.00 & $0.26(0.07-1.04)$ \\
\hline Model 4 & 1.00 & $0.25(0.06-1.03)$ \\
\hline Model 5 & 1.00 & $0.24(0.06-0.98)$ \\
\hline
\end{tabular}

Model 1: adjusted for age and sex

Model 2: additionally adjusted for region and BMI

Model 3: additionally adjusted for smoking, physical activity, alcohol consumption

Model 4: additionally adjusted for kidney disease, antihypertensive and cholesterol lowering drug use

Model 5: additionally adjusted for blood DHA 
serum potassium; by contrast, $\mathrm{Mg}$ can attenuate arrhythmias regardless of $\mathrm{sMg}$ concentration [30,32,33]. Irrespective of the degree to which prevalence of PVC may differ due to sMg concentration independent of other electrolytes, this study supports hypomagnesemia as a risk factor for ventricular arrhythmias.

\section{Magnesium \& arrhythmias: T2DM as an effect modifier}

Low serum Mg has been associated with T2DM [34], but not metabolic states preceding T2DM $[35,36]$, a finding consistent with our data. In this sample, diabetic status, but not MetS, was a major determinant of very low sMg levels; $76 \%$ of all hypomagnesemic adults had T2DM. While increased fractional excretion of $\mathrm{Mg}$ at elevated insulin concentrations [37] may be at play in both MetS and T2DM, mechanisms for Mg loss specific to diabetes, such as renal $\mathrm{Mg}$ wasting secondary to osmotic diuresis during glucosuria [38], might be associated with comparatively greater reductions in $\mathrm{sMg}$.

As T2DM is the most common condition associated with low sMg [15] and diabetes significantly increases risk of ventricular arrhythmias [16], there is theoretical basis for the hypothesis that T2DM might modify the association between $\mathrm{sMg}$ and PVC. Here, we provide the first supporting evidence that T2DM is an important effect modifier of the association between $\mathrm{sMg}$ and PVC, with reductions in PVC prevalence across the $\mathrm{sMg}$ gradient in T2DM only, driving the significant associations observed in the entire population. While the PVC outcome variable ( $>30 \mathrm{PVC} / \mathrm{hr}$, multiform or repetitive) in the Framingham Offspring cohort was different than endpoints assessed here and T2DM prevalence was high in our population, whether the significant association between sMg and PVC risk reported in the Framingham cohort [14] might be modified by inclusion of T2DM status as a covariate is unknown. Replication studies and explicit testing for potential effect modification by diabetes in future analyses is required.

In this work, risk of $>6 \mathrm{PVC} / \mathrm{hr}$ was maximally reduced above a $\mathrm{sMg}$ threshold $(0.75 \mathrm{mmol} / \mathrm{L})$ within the normal sMg concentration range and prevalence of PVC declined across the normal sMg concentration gradient in adults with T2DM. In other populations, significantly reduced risk of ventricular arrhythmia [14], sudden cardiac death [39] and cardiovascular disease death $[40,41]$ at elevated sMg concentrations have been reported. Thus, the findings of this study are consistent with evidence from other cross-sectional and prospective cohort studies reporting cardiovascular risk stratification within the normal sMg range, with reduced risk of both intermediary adverse outcomes and hard endpoints at higher sMg concentrations.

\section{Limitations}

Limitations of this study include use of sMg instead of ionized $\mathrm{Mg}$ as a biomarker [26]; free intracellular $\mathrm{Mg}$ is the fraction regulating enzyme pathways [27]. Although sMg may not be a sensitive marker in reflecting mild $\mathrm{Mg}$ inadequacy or the intracellular $\mathrm{Mg}$ pool, further investigations evaluating the utility of $\mathrm{sMg}$, an inexpensive and simple measure, as a cardiovascular risk biomarker are appropriate.

Consistent cut-offs for the number or complexity (multiforms, pairs, runs, R-on-T) of PVC per unit time of Holter recording associated with mortality risk across population groups of different ages, ethnicities or underlying cardiovascular risk have yet to be established [3-6]. Thus, a main limitation of this work is the uncertain prognostic significance of PVC endpoints selected in this high-risk population, which can be determined through long term follow-up for incidence of ventricular fibrillation, tachycardia, sudden cardiac death, or CVD mortality. Other limitations include the use of shortterm $(2 \mathrm{hr})$ Holter monitoring on a single occasion for PVC detection, and unknown left ventricular ejection fraction, the latter of which could have modulated prevalence of asymptomatic heart disease and PVC among patients with diabetes. Generalizability of our findings may be limited by the ethnic homogeneity of the sample and high prevalence of T2DM relative to most other general populations. Inclusion of other potentially relevant unmeasured covariates, such as serum potassium and other electrolytes, caffeine use, non-cardiovascular drugs, or stress/anxiety might reduce residual confounding. Consistency in sample demographics and CVD risk, monitoring environments, duration and specific PVC endpoints assessed in different populations will be important in reducing heterogeneity among future studies and facilitating comparisons.

\section{Conclusions}

This study provides evidence that T2DM is an important effect modifier of the association between sMg and ventricular ectopy, with significant reductions in PVC prevalence across the $\mathrm{sMg}$ gradient in adults with T2DM. Diabetic status, but not MetS, was a major determinant of very low sMg levels in this population. PVC risk was particularly elevated in diabetic adults with hypomagnesemia, suggesting that future interventions to increase $\mathrm{sMg}$ concentrations among adults with T2DM may confer protection against ventricular arrhythmias. As hypomagnesemia is an under-diagnosed electrolyte abnormality common in T2DM [42], appropriately powered randomized controlled clinical trials are required to evaluate the potential of $\mathrm{Mg}$ to reduce arrhythmias and cardiovascular risk. 


\section{Additional material}

Additional file 1: Adjusted odds ratios and 95\% confidence intervals for presence of $>6 \mathrm{PVC} / \mathrm{hr}$ on Holter monitors across the sMg concentration range $(n=750)$.

Additional file 2: Odds ratios for the fully adjusted model (Model 5) for presence of $>6 \mathrm{PVC} / \mathrm{hr}$ on Holters are minimized using $<0.75$ $\mathrm{mmol} / \mathrm{L} \mathrm{sMg}$ as the reference $(\mathrm{n}=750)$.

\begin{abstract}
Abbreviations
ACC: American College of cardiology; AHA: American heart association; ARIC: Atherosclerosis risk in communities; BMI: Body mass index; CVD: Cardiovascular event or conditions; $\mathrm{Cl}$ : Confidence interval; CBHSSJB: Cree board of health and social services of James bay; DHA: Docosahexaenoic acid; EPA: Eicosapentaenoic acid; ESC: European society of cardiology; FG: Fasting glucose; HR: Hazard ratio; IDF: International diabetes federation; Mg: Magnesium; MetS: Metabolic syndrome; OR: Odds ratio; PVC: Premature ventricular complexes; sMg: Total serum magnesium; T2DM: Type 2 diabetes mellitus; USDA: United states department of agriculture.
\end{abstract}

\section{Acknowledgements}

The authors wish to thank lab managers Donna Leggee and Suzanne Cote. This scientific communication is a report from the Nituuchischaayihtitaau Aschii: Multi-Community Environment-and-Health Longitudinal Study in liyiyiu Aschii supported by the Cree people of northern Québec, the Cree First Nations and the Cree Board of Health and Social Services of James Bay.

\section{Author details}

${ }^{1}$ School of Dietetics \& Human Nutrition, McGill University, 21,111 Lakeshore Road, Ste. Anne de Bellevue, Quebec, H9X 3V9, Canada. '2Division of Preventive Medicine, Brigham and Women's Hospital, 900 Commonwealth Ave., Boston, Massachusetts, 02215, USA. ${ }^{3}$ Quebec Heart and Lung Institute, Sainte-Foy, Quebec, Canada. ${ }^{4}$ Public Health Research Unit, CHUL Research Center, Centre Hospitalier, Universitaire de Quebec Sainte-Foy, Quebec City, Quebec, G1V 5B3, Canada. ${ }^{5}$ Department of Pathology and Laboratory Medicine, University of Louisville, Louisville, Kentucky, USA 40292.

\section{Authors' contributions}

ED and GE conceived and designed the Cree health study and contributed to critical review of analysis and interpretation of data; YS, PP and RE critically reviewed the statistical design, analysis and interpretation of data; LD conducted the statistical analysis, drafted the manuscript and was responsible for its content. All authors read and approved the final manuscript.

\section{Competing interests}

The authors declare that they have no competing interests.

Received: 9 February 2012 Accepted: 9 March 2012

Published: 9 March 2012

\section{References}

1. Kennedy HL, Whitlock JA, Sprague MK, Kennedy LJ, Buckingham TA, Goldberg RJ: Long-term follow-up of asymptomatic healthy subjects with frequent and complex ventricular ectopy. N Engl J Med 1985, 312:193-197.

2. Gaita F, Giustetto C, Di Donna P, Richiardi E, Libero L, Rosa MC, Molinari G, Trevi G: Long-term follow-up of right ventricular monomorphic extrasystoles. J Am Coll Cardiol 2001, 38:364-370.

3. Sajadieh A, Nielsen OW, Rasmussen V, Hein HO, Frederiksen BS, Davanlou M, Hansen JF: Ventricular arrhythmias and risk of death and acute myocardial infarction in apparently healthy subjects of age $>=55$ years. Am J Cardiol 2006, 97:1351-1357.

4. Cheriyath P, He F, Peters I, Li XA, Alagona P, Wu CT, Pu M, Cascio WE, Liao DP: Relation of atrial and/or ventricular premature complexes on a two-minute rhythm strip to the risk of sudden cardiac death (the atherosclerosis risk in communities [ARIC] study. Am J Cardiol 2011, 107:151-155
5. Stein PK, Sanghavi D, Sotoodehnia N, Siscovick DS, Gottdiener J: Association of Holter-based measures including T-wave altemans with risk of sudden cardiac death in the community-dwelling elderly: the cardiovascular health study. J Electrocardiol 2010, 43:251-259.

6. Abdalla ISH, Prineas RJ, Neaton JD, Jacobs DR, Crow RS: Relation between ventricular premature complexes and sudden cardiac death in apparently healthy men. Am J Cardiol 1987, 60:1036-1042.

7. Raiha IJ, Piha SJ, Seppanen A, Puukka P, Sourander L: Predictive value of continuous ambulatory electrocardiographic monitoring in elderly people. Br Med J 1994, 309:1263-1267.

8. Fawcett WJ, Haxby EJ, Male DA: Magnesium: physiology and pharmacology. Br J Anaesth 1999, 83:302-320.

9. Abbot LG, Rude RK: Clinical manifestations of magnesium deficiency. Miner Electrolyte Metab 1993, 19:314-322.

10. Parikka H, Toivonen L, Naukkarinen V, Tierala I, Pohjola-Sintonen S, Heikkila J, Nieminen MS: Decreases by magnesium of QT dispersion and ventricular arrhythmias in patients with acute myocardial infarction. Eur Heart J 1999, 20:111-120.

11. Chakraborti S, Chakraborti T, Mandal M, Mandal A, Das S, Ghosh S: Protective role of magnesium in cardiovascular diseases: a review. $\mathrm{Mol}$ Cell Biochem 2002, 238:163-179.

12. Nielsen FH, Milne DB, Klevay LM, Gallagher S, Johnson L: Dietary magnesium deficiency induces heart rhythm changes, impairs glucose tolerance, and decreases serum cholesterol in post menopausal women. J Am Coll Nutr 2007, 26:121-132.

13. Klevay LM, Milne DB: Low dietary magnesium increases supraventricular ectopy. Am J Clin Nutr 2002, 75:550-554

14. Tsuji H, Venditti FJ, Evans JC, Larson MG, Levy D: The associations of levels of serum potassium and magnesium with ventricular premature complexes (The Framingham heart study). Am J Cardiol 1994, 74:232-235.

15. Rude RK, Shils ME: Magnesium. In Modern Nutrition in Health and Disease.. 10 edition. Edited by: Shils ME, Shike M, Ross AC, Caballero B, Cousins RJ. New York: Lippincott Williams 2006:234.

16. Escobedo LG, Caspersen CJ: Risk factors for sudden coronary death in the United States. Epidemiol 1997, 8:175-180.

17. Nituuchischaayihtitaau Aschii Multi-community Environment-and-health. Longitudinal Study in liyiyiu Aschii: Mistissini technical report: summary of activities, results and recommendations. [http://www.creehealth.org/ sites/default/files/Environmental\%20Health\%20Study-\%20Technical\% 20Report\%20of\%20Mistissini.pdf].

18. Harris SB, Gittelsohn J, Hanley A, Barnie A, Wolever TM, Gao XJ, Logan A Zinman B: The prevalence of NIDDM and associated risk factors in native Canadians. Diabetes Care 1997, 20:185-187.

19. Egeland GM, Denomme D, Lejeune P, Pereg D: Concurrent validity of the international physical activity questionnaire (IPAQ) in an liyiyiu Aschii (Cree) community. Can J Pub Health 2008, 99:307-310.

20. Russo GL: Dietary $n-6$ and $n-3$ polyunsaturated fatty acids: from biochemistry to clinical implications in cardiovascular prevention. Biochem Pharmacol 2009, 77:937-946.

21. Mozaffarian D, Psaty BM, Rimm EB, Lemaitre RN, Burke GL, Lyles MF, Lefkowitz D, Siscovick DS: Fish intake and risk of incident atrial fibrillation. Circulation 2004, 110:368-373.

22. Alberti KGMM, Ecklel RH, Grundy SM, IDF Epidemiology Task Force Consensus Group: Harmonizing the metabolic syndrome. Circulation 2009, 120:1640-1645.

23. Stein PK, Barzilay Jl, Chaves PHM, Mistretta SQ, Domitrovich PP, Gottdiener JS, Rich MW, Kleiger RE: Novel measures of heart rate variability predict cardiovascular mortality in older adults independent of traditional cardiovascular risk factors: the cardiovascular health study (CHS). J Cardio Electrophysiol 2008, 19:1169-1174.

24. Alghamdi SMG, Cameron EC, Sutton RAL: Magnesium deficiency: pathophysiologic and clinical overview. Am J Kidney Dis 1994, 24:737-752.

25. Loeb HS, Pietras RJ, Gunnar RM, Tobin JR: Paroxysmal ventricular fibrillation in two patients with hypomagnesemia. Circulation 1968, 3:210-215.

26. Dyckner T, Wester PO: Magnesium deficiency contributing to ventricular tachycardia. Acta Med Scand 1982, 212:89-91.

27. Ramme SP, White CJ, Svinarich JT, Watson TD, Fox RF: Torsade de pointes and magnesium deficiency. Am Heart J 1985, 109:89-91.

28. Zipes DP, Camm AJ, Borggrefe M, Buxton AE, Chaitman B, Fromer M, Gregoratos G, Klein G, Moss AJ, Myerburg RJ, Priori SG, Quinones MA, 
Roden DM, Silka MJ, Tracy C, Smith SC Jr, Jacobs AK, Adams CD, Antman EM, Anderson JL, Hunt SA, Halperin JL, Nishimura R, Ornato JP, Page RL, Riegel B, Blanc JJ, Budaj A, Dean V, Deckers JW, et al: ACC/AHA/ ESC 2006 guidelines for management of patients with ventricular arrhythmias and the prevention of sudden cardiac death: a report of the American College of cardiology/American heart association task force and the European Society of cardiology committee for practice guidelines (Writing committee to develop guidelines for management of patients with ventricular arrhythmias and the prevention of sudden cardiac death). J Am Coll Cardiol 2006, 48:e247-e346.

29. Belardinelli L, Antzelevitch C, Vos MA: Assessing predictors of druginduced torsade de pointes. Trends Pharmacol Sci 2003, 24:619-625.

30. Millante TA, Ward DE, Camm AJ: Is hypomagnesemia arrhythmogenic? Clin Cardiol 1992, 15:103-108.

31. Mubagwa K, Gwanyanya A, Zakharov S, Macianskiene R: Regulation of cation channels in cardiac and smooth muscle cells by intracellular magnesium. Arch Biochem Biophys 2007, 458:73-89.

32. Harris I, Levin D: The effects upon the human electrocardiogram of the introduction of calcium and potassium into the blood. J Physiol 1937, 89:153.

33. Saran T, Perkins JD, Javed MA: Does the prophylactic administration of magnesium sulphate to patients undergoing thoracotomy prevent postoperative supraventricular arrhythmias? A randomized controlled trial. Br J Anaesth 2011, 106:785-791.

34. Håglin L, Backman L, Tornkvist BL: A structural equation model for assessment of links between changes in serum triglycerides, -urate, and -glucose and changes in serum calcium, -magnesium and -phosphate in type 2 diabetes and non-diabetes metabolism. Cardiovasc Diabetol 2011, 10:116.

35. Simmons $D$, Joshi S, Shaw J: Hypomagnesaemia is associated with diabetes: not pre-diabetes, obesity or the metabolic syndrome. Diabetes Res Clin Pract 2010, 87:261-266.

36. Røislien J, Van Calster B, Hjelmesæth J: Parathyroid hormone is a plausible mediator for the metabolic syndrome in the morbidly obese: a crosssectional study. Cardiovasc Diabetol 2011, 10:17.

37. Kalaitzidis R, Tismihodimos V, Bairaktari E, Siamopoulos K, Elisaf M: Disturbances of phosphate metabolism: another feature of the metabolic syndrome. J Kidney Dis 2005, 45:851-858.

38. de Valk HW: Magnesium in diabetes mellitus. Neth J Med 1999, 54:139-146.

39. Peacock JM, Ohira T, Post W, Sotoodehnia N, Rosamond W, Folson AR: Serum magnesium and risk of sudden cardiac death in the atherosclerosis risk in communities (aric) study. Am Heart J 2010, 160:464-470.

40. Reffelmann T, Ittermann T, Dörr M, Völzke H, Reinthaler M, Petersmann A, Felix SB: Low serum magnesium concentrations predict cardiovascular and all-cause mortality. Atherosclerosis 2011, 219:280-284.

41. Ford ES: Serum magnesium and ischaemic heart disease: findings from a national sample of US adults. Int J Epidemiol 1999, 28:645-651.

42. Barbagallo M, Dominguez LJ: Magnesium metabolism in type 2 diabetes mellitus, metabolic syndrome and insulin resistance. Arch Biochem Biophys 2007, 458:40-47.

doi:10.1186/1475-2840-11-23

Cite this article as: Del Gobbo et al:: Low serum magnesium

concentrations are associated with a high prevalence of premature ventricular complexes in obese adults with type 2 diabetes.

Cardiovascular Diabetology 2012 11:23.

\section{Submit your next manuscript to BioMed Central and take full advantage of:}

- Convenient online submission

- Thorough peer review

- No space constraints or color figure charges

- Immediate publication on acceptance

- Inclusion in PubMed, CAS, Scopus and Google Scholar

- Research which is freely available for redistribution 